\title{
GRASSMANN SEMIALGEBRAS AND THE CAYLEY-HAMILTON THEOREM
}

\author{
LETTERIO GATTO AND LOUIS ROWEN
}

(Communicated by Jerzy Weyman)

\begin{abstract}
We develop a theory of Grassmann semialgebra triples using HasseSchmidt derivations, which formally generalizes results such as the CayleyHamilton theorem in linear algebra, thereby providing a unified approach to classical linear algebra and tropical algebra.
\end{abstract}

\section{INTRODUCTION}

The main goal of this paper is to define and explore the semiring version of the theory [4 6] of the first author concerning the Grassmann exterior algebra, viewed more generally in terms of negation maps and systems, continuing the approach of 21. This provides a robust structure which unifies and generalizes four major versions of Grassmann structures, cf. Note 1.1. and provides a generalization of the Cayley-Hamilton theorem in Theorem 3.15. In the process we investigate HasseSchmidt derivations on Grassmann systems. The version given in Theorem 2.4 (over a free module $V$ over an arbitrary semifield) is the construction which seems to "work".

Generalizing negation in Definition 1.3 to the notion of a "negation map" (-) satisfying all the usual properties of negation except $a(-) a=0$ (studied in [21]), we find that the Grassmann semialgebra of a free module $V$, described in Theorem 2.4, has a natural negation map on all homogeneous vectors, with the ironic exception of $V$ itself, obtained by switching two tensor components.

Theorem A (Theorem 2.4). $\bigoplus_{n \geq 2} V^{\otimes(n)}$ has a negation map (-) satisfying

$$
b_{\pi\left(i_{1}\right)} \cdots \bar{b}_{\pi\left(i_{t}\right)}=(-)^{\pi} b_{i_{1}} \cdots \bar{b}_{i_{t}}
$$

for $b_{i_{j}} \in V$.

Received by the editors May 14, 2018, and, in revised form, May 13, 2020.

2020 Mathematics Subject Classification. Primary 15A75, 16Y60, 15A18; Secondary 12K10, $14 \mathrm{~T} 10$.

Key words and phrases. Cayley-Hamilton theorem, exterior semialgebras, Grassmann semialgebras, Hasse-Schmidt derivations, differentials, eigenvalues, eigenvectors Laurent series, Newton's formulas, power series, semifields, systems, semialgebras, tropical algebra, triples.

The first author was partially supported by INDAM-GNSAGA and by PRIN "Geometria sulle varietà algebriche" Progetto di Eccellenza Dipartimento di Scienze Matematiche, 2018-2022 no. E11G18000350001.

The second author was supported in part by the Israel Science Foundation, grant No. 1207/12 and his visit to Torino was supported by the "Finanziamento Diffuso della Ricerca", grant no. 53_RBA17GATLET, of Politecnico di Torino. 
This provides us "enough" negation, coupled with the relation $\preceq$ 。 of Definition 1.6 (designed to replace equality), to adapt the methods of [6] to negation maps and systems of [21] to study $T(V)$, with $\mathcal{T}$ the nonzero simple tensors.

One obtains a Grassmann algebra by modding out all elements of the form $v \otimes v$, $v \in V$. A weaker version is obtained when we take a given base $\left\{b_{0}, b_{1}, \ldots, b_{n-1}\right\}$ of $V$ and mod only the $b_{i} \otimes b_{i}, 0 \leq i<n$. One can enhance these by means of a "symmetrizing" construction which provides a negation map and a triple for any Grassmann algebra:

Theorem B (Theorems 2.13, 2.14, 2.22). There $i s$ a symmetrized triple $\left(\mathfrak{G}(V)^{\natural}, \widehat{\mathcal{T}},(-)_{\mathrm{sw}}\right)$, together with an embedding of triples $\left(\mathfrak{G}(V)_{\geq 2}, \mathcal{T}_{\mathfrak{G}(V)_{\geq 2}},(-)\right) \rightarrow$ $\left(\mathfrak{G}(V)^{\natural}, \widehat{\mathcal{T}},(-)_{\mathrm{sw}}\right)$ given by $c \mapsto(c, 0)$. An analogous assertion holds for $\mathfrak{G}(V)^{\diamond}$.

Note 1.1. There are four major applications of this Grassmann triple:

(i) The classical situation is recovered, where $V$ is a vector space and (-) is the classical negation.

(ii) The tropical situation is treated in 8 to study matroids, taken over the max-plus algebra, cf. Remark 2.26 where $(-)$ is the identity map.

(iii) The supertropical situation, as described in Theorem 2.13. provides an alternate language for the theory of [8].

(iv) The symmetrized situation, as described in 2.1 provides a nontrivial negation map for any Grassmann semialgebra.

The rest of 92 is dedicated to laying the groundwork for further research in Grassmann semialgebras, and the reader only interested in the applications of this paper could go on to 93 where we study properties of derivations. There we learn how to associate to an endomorphism $f$ of an $\mathcal{A}$ free module $V_{n}$ a HasseSchmidt derivation $D\{z\}: \bigwedge V_{n} \rightarrow \bigwedge V_{n}[[z]]$, on the Grassmann semi-algebra, i.e $D\{z\}(u \wedge v)=D\{z\} u \wedge D\{z\} V$ and $D\{z\}_{\left.\right|_{V_{n}}}=\sum_{j \geq 0} f_{j} z^{j}$. We suitably construct, starting from the data of $D\{z\}$, an operator valued polynomial $\bar{D}\{z\}: \bigwedge V_{n} \rightarrow$ $\operatorname{End}\left(\bigwedge V_{n}\right)[z]$ showing in detail:

Theorem C (Theorem 3.12). The polynomial $\bar{D}\{z\}$ is a quasi-inverse of $D\{z\}$, in the sense that $\bar{D}\{z\} D\{z\} u \succeq u$ for all $u \in \wedge V_{n}$.

Our main result, Theorem 3.14 describes the relation between a Hasse-Schmidt derivation $D\{z\}$ and its "quasi-inverse" $\bar{D}\{z\}$ defined in such a way to yield:

Theorem D (Theorem 3.14). $\bar{D}\{z\}(D\{z\} u \wedge v) \succeq u \wedge \bar{D}\{z\} v$.

In the classical case, one gets equality as shown in Remark 3.16, so we recover [6]. Our main application in this paper is a generalization of the Cayley-Hamilton theorem to semi-algebras.

Theorem E (Theorem 3.15).

$$
\left(\left(D_{n} u+e_{1} D_{n-1} u+\cdots+e_{n} u\right) \wedge v\right)(-)\left(\left(D_{n} u+e_{1}^{\prime} D_{n-1} u+\cdots+e_{n}^{\prime} u\right) \wedge v\right) \succeq 0
$$

for all $u \in \bigwedge^{>0} V_{n}$.

We obtain true equality in these theorems by modding out all elements of the form $v \otimes v$. Again we recover [6] in the classical case. 
1.1. Basic notions. Much of this section is a review of [21], as summarized in 22], and also as in [15]. As customary, $\mathbb{N}$ denotes the natural numbers including $0, \mathbb{N}^{+}$ denotes $\mathbb{N} \backslash\{0\}, \mathbb{Q}$ denotes the rational numbers, and $\mathbb{R}$ denotes the real numbers, all ordered monoids under addition.

A semiring ${ }^{\dagger \dagger}(\mathcal{A},+, \cdot)$ is an additive abelian semigroup $(\mathcal{A},+)$ and multiplicative semigroup $(\mathcal{A}, \cdot)$ satisfying the usual distributive laws. A semiring ${ }^{\dagger}(\mathcal{A},+, \cdot, \mathbb{1})$ is a semiring ${ }^{\dagger \dagger}$ with a multiplicative unit $\mathbb{1}$. (Thus, an ideal of a semiring ${ }^{\dagger}$ is a

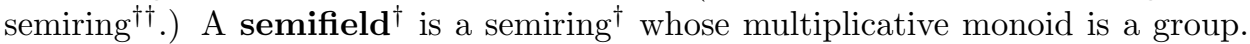
A semiring (resp. semifield) [9] is a semiring ${ }^{\dagger}$ (resp. semifield ${ }^{\dagger}$ ) with an absorbing element $\mathbb{0}$ formally adjoined.

Definition 1.2. A $\mathcal{T}$-module over a set $\mathcal{T}$ is an additive monoid $(\mathcal{A},+, \mathbb{O})$ with a scalar multiplication $\mathcal{T} \times \mathcal{A} \rightarrow \mathcal{A}$ satisfying the following axioms, $\forall k \in \mathbb{N}, a \in \mathcal{T}$, $b, b_{j} \in \mathcal{A}$ :

(i) (Distributivity over $\mathcal{T}): a\left(\sum_{j=1}^{k} b_{j}\right)=\sum_{j=1}^{k}\left(a b_{j}\right)$.

(ii) $a \mathbb{0}_{\mathcal{A}}=\mathbb{O}_{\mathcal{A}}$.

A $\mathcal{T}$-monoid module over a multiplicative monoid $\mathcal{T}$ is a $\mathcal{T}$-module satisfying the extra conditions

$$
\mathbb{1}_{\mathcal{T}} b=b, \quad\left(a_{1} a_{2}\right) b=a_{1}\left(a_{2} b\right), \quad \forall a_{i} \in \mathcal{T}, b \in \mathcal{A} .
$$

A $\mathcal{T}$-semiring is a semiring that is also a $\mathcal{T}$-monoid module over a given multiplicative submonoid $\mathcal{T}$. This paper only concerns $\mathcal{T}$-semirings, which are closely related to blueprints in [19. We put $\mathcal{T}_{\mathbb{D}}=\mathcal{T} \cup\{\mathbb{0}\}$.

Tensor products over semirings [17, 18,23, are analogous to tensor products over rings. The tensor product $\mathcal{M}_{1} \otimes_{\mathcal{A}} \mathcal{M}_{2}$ of right and left $\mathcal{A}$-modules $\mathcal{M}_{1}$ and $\mathcal{M}_{2}$ is $\left(\mathcal{F}_{1} \oplus \mathcal{F}_{2}\right) / \Phi$, where $\mathcal{F}_{i}$ is the free module (respectively right or left) with base $\mathcal{M}_{i}$, and $\Phi$ is the congruence generated by all

$$
\begin{gathered}
\left(\left(\sum_{j} x_{1, j}, \sum_{k} x_{2, k}\right), \sum_{j, k}\left(x_{1, j}, x_{2, k}\right)\right) \\
\left(\left(x_{1} a, x_{2}\right),\left(x_{1}, a x_{2}\right)\right) \quad \forall x_{i}, x_{i, j}, x_{i, k} \in \mathcal{M}_{i}, a \in \mathcal{A} .
\end{gathered}
$$

\subsection{Negation maps, triples, and systems.}

Definition 1.3. A negation map on a $\mathcal{T}$-module $M$ over a given set $\mathcal{T}$ is a semigroup isomorphism (-) $: M \rightarrow M$ of order $\leq 2$, written $b \mapsto(-) b$, which also respects the $\mathcal{T}$-action in the sense that

$$
(-)(a b)=a((-) b)
$$

for $a \in \mathcal{T}, b \in M$.

A semiring ${ }^{\dagger \dagger}$ negation map on a semiring ${ }^{\dagger \dagger} \mathcal{A}$ is a negation map which satisfies $(-)(a b)=a((-) b)=((-) a) b$ for all $a, b \in \mathcal{A}$.

In the classical case the negative is a negation map. For tropical algebra, one could just take $(-)$ to be the identity map, but we want a less trivial example.

We write $a(-) b$ for $a+((-) b),( \pm) a$ for $\{a,(-) a\}$, and $a^{\circ}$ for $a(-) a$, called a quasi-zero. The set $M^{\circ}$ of quasi-zeroes is an important $\mathcal{T}$-submodule of $M$. When $\mathcal{A}$ is a semiring, $\mathcal{A}^{\circ}$ is an ideal.

We define $(-)^{\mathbb{D}} a$ to be $a$ and, for $k \in \mathbb{N}$, we inductively define $(-)^{k} a$ to be $(-)\left((-)^{k-1} a\right)$. 
Lemma 1.4. $\left((-)^{k} a\right)\left((-)^{k^{\prime}} a^{\prime}\right)=(-)^{k+k^{\prime}}\left(a a^{\prime}\right)$ for $a, a^{\prime} \in \mathcal{A}$.

Proof. $\left((-)^{k} a\right)\left((-)^{k^{\prime}} a^{\prime}\right)=(-)\left((-)^{k-1} a\right)\left((-)^{k^{\prime}} a^{\prime}\right)=(-)\left((-)^{k+k^{\prime}-1}\left(a a^{\prime}\right)\right)$ $=(-)^{k+k^{\prime}}\left(a a^{\prime}\right)$, by induction on $k$.

Definition 1.5. A pseudo-triple is a collection $(\mathcal{A}, \mathcal{T},(-))$, where $(-)$ is a negation map on both $\mathcal{T}$ and $\mathcal{A}$, and $\mathcal{A}$ is a $(\mathcal{T},(-))$-module.

A $\mathcal{T}_{\mathcal{A}}$-pseudo-triple $\left(\mathcal{A}, \mathcal{T}_{\mathcal{A}},(-)\right)$ is a $\mathcal{T}$-module $\mathcal{A}$, with $\mathcal{T}_{\mathcal{A}}$ designated as a distinguished subset, and a negation map (-) satisfying $(-) \mathcal{T}_{\mathcal{A}}=\mathcal{T}_{\mathcal{A}}$. A $\mathcal{T}_{\mathcal{A}}$-triple, called a triple when $\mathcal{T}$ is understood, is a $\mathcal{T}_{\mathcal{A}}$-pseudo-triple, in which $\mathcal{T}_{\mathcal{A}} \cap \mathcal{A}^{\circ}=\emptyset$ and $\mathcal{T}_{\mathcal{A}}$ generates $(\mathcal{A} \backslash\{\mathbb{O}\},+)$.

Since $\mathcal{T}_{\mathcal{A}}$ is usually clear from the context, we abuse notation and write $\mathcal{T}$ for $\mathcal{T}_{\mathcal{A}} \subseteq \mathcal{A}$. A triple is uniquely negated when for any $a \in \mathcal{T}, a+b \in \mathcal{A}^{\circ}$ implies $b=(-) a$.

The structure is rounded out with the following relation.

Definition 1.6. Define the o-surpassing relation $\preceq_{\circ}$ on a module $M$ with negation map by $a_{0} \preceq_{\circ} a_{1}$ if $a_{1}=a_{0}+d$ for some $d \in M^{\circ}$.

A uniquely negated triple $(\mathcal{A}, \mathcal{T},(-))$ together with a surpassing relation $\preceq$ is called a system 1

Remark 1.7. The relation $\preceq$ 。 on a system restricts to equality on $\mathcal{T}$, by [21, Proposition 4.4]. In fact $\preceq_{\circ}$ is used to replace equality when we work with triples, and identities in classical algebra can often be replaced by relations expressed in terms of $\preceq_{0}$, by means of the transfer principle of [1], formulated for systems in [21, Theorem 6.17].

1.3. Functions to $\mathcal{A}$. The next construction, discussed in [16, §4.2], enables us to describe power series in a structural context. From now on, we suppose $(S,+)$ is a semigroup, often $(\mathbb{N},+, 0)$. Given a triple $(\mathcal{A}, \mathcal{T},(-)), \mathcal{A}^{S}$ denotes the maps from $S$ to $\mathcal{A}$, and $\mathcal{T}^{S}$ denotes the nonzero maps of $\mathcal{A}^{S}$ sending $S$ to $\mathcal{T}$. For example, for $c \in \mathcal{A}$, the constant function $\tilde{c}$ is given by $\tilde{c}(s)=c$ for all $s \in S$.

We modify the definition of support from [16, Definition 4.2].

Definition 1.8. Given $f \in \mathcal{A}^{S}$ we define its support $\operatorname{supp}(f):=\{s \in S: f(s) \neq$ O) , and $\operatorname{supp}\left(\mathcal{A}^{S}\right)$ for $\cup\left\{\operatorname{supp}(f): f \in \mathcal{A}^{S}\right\}$.

Definition 1.9. A set $\mathcal{A}$ b of maps $f: S \rightarrow \mathcal{A}$ is convolution admissible if for each $f, g \in \mathcal{A} b$ and $s \in S$ there are only finitely many $s^{\prime} \in \operatorname{supp}(f), s^{\prime \prime} \in \operatorname{supp}(g)$, with $s^{\prime}+s^{\prime \prime}=s$.

Example 1.10. $\mathcal{A} b$ is convolution admissible whenever $S=\mathbb{N}^{(I)}$ (the direct sum) for some index set $I$, since the condition of Definition [1.9 already holds in $S$.

Definition 1.11. Suppose $\mathcal{A} b$ is a convolution admissible set. The convolution product $\mathcal{A} b \times \mathcal{A} b \rightarrow \mathcal{A} b$ is given by defining $f g$ to be the function satisfying

$$
f g(s)=\sum_{s^{\prime}+s^{\prime \prime}=s} f\left(s^{\prime}\right) g\left(s^{\prime \prime}\right) .
$$

\footnotetext{
${ }^{1}$ In [16], in the systemic setting, a more general notion of surpassing map $\preceq$ is used, and $\mathcal{A}_{\mathrm{Null}}$ is introduced which equals $\mathcal{A}^{\circ}$ when $\preceq=\preceq \circ$; here, using Lemma 2.18 as justification, we use $\mathcal{A}^{\circ}$ and $\preceq=\preceq$ 。 to avoid complications.
} 
The intuitive way to receive a negation map on $\mathcal{A}$ b from a negation map on $\mathcal{A}$ would be to define $((-) f)(s)=(-)(f(s))$; these maps also are convolution admissible, so one would expand $\mathcal{A} b$ to include them.

1.4. Graded semirings and modules. We want to grade semirings and their modules. We define direct sums in the usual way.

Definition 1.12. An $L$-graded $\mathcal{T}$-semiring ${ }^{\dagger}$ is a $\mathcal{T}$-semiring ${ }^{\dagger} \mathcal{R}$ which also is an $L$-graded $\mathcal{T}$-module $\mathcal{R}:=\oplus_{\ell \in L} \mathcal{R}_{\ell}$ for semigroups $\left(\mathcal{R}_{\ell},+\right)$ satisfying the following conditions, where $\mathcal{T}_{\ell}=\mathcal{T} \cap \mathcal{R}_{\ell}$ :

(i) $\mathcal{T}=\cup_{\ell \in L} \mathcal{T}_{\ell}$;

(ii) $\mathcal{R}_{\ell} \mathcal{R}_{\ell^{\prime}} \subseteq \mathcal{R}_{\ell+\ell^{\prime}}, \forall \ell, \ell^{\prime} \in L$.

Note that $\mathcal{R}_{\mathbb{0}}$ is a $\mathcal{T}_{\mathbb{D}}$-module, and also a semiring ${ }^{\dagger}$, over which each $\mathcal{R}_{\ell} \cup\{\mathbb{O}\}$ is a module.

When we turn to Grassmann semialgebras, $L$ will be ordered with a minimal element 0 ; one could take $L=\mathbb{N}$, for example. We write $\mathcal{M}^{>0}:=\oplus_{\ell>0} \mathcal{M}_{\ell}$, a submodule of $\mathcal{M}$ lacking the constant component. Then $\mathcal{R}^{>0}$ is a sub-semiring ${ }^{\dagger \dagger}$ of $\mathcal{R}$.

1.4.1. Super-semialgebras. Here is an interesting special case.

Definition 1.13. A super-semialgebra is a $\mathbb{Z}_{2}$-graded semialgebra $\mathcal{A}:=\mathcal{A}_{0} \oplus \mathcal{A}_{1}$, i.e., satisfying twist multiplication:

$$
\left(a_{0}, a_{1}\right)\left(a_{0}^{\prime}, a_{1}^{\prime}\right)=\left(a_{0} a_{0}^{\prime}+a_{1} a_{1}^{\prime}, a_{0} a_{1}^{\prime}+a_{1} a_{0}^{\prime}\right) .
$$

A natural way of getting a $\mathbb{Z}_{2}$-grade from an $\mathbb{N}$-graded semialgebra is to take the 0 -grade to be the set of even indices and the 1-grade to be the set of odd indices.

1.4.2. The power series semiring of a graded semiring ${ }^{\dagger}$. From now on, we take $S=\mathbb{N}$ as in Example 1.10, which is equivalent to the following.

Definition 1.14. Given an $\mathbb{N}$-graded $\mathcal{T}$-semiring $\mathcal{R}$ with respect to the semigroup $(\mathbb{N},+)$, we define the power series semiring $\mathcal{R}[[z]]$ over a central indeterminate $z$, in the usual way as possibly infinite formal sums (with convolution product), and its sub-semiring $\mathcal{R}[z]=\sum_{j} \mathcal{R}_{j} z^{j}$.

Lemma 1.15. $\mathcal{R}[[z]]$ and $\mathcal{R}[z]$ are indeed semirings. Both $\mathcal{R}[[z]]$ and $\mathcal{R}[z]$ are graded by the powers of $z$.

Proof. $\mathcal{R}[[z]]$ satisfies the axioms of a semiring, by the customary verification, and its subset $\mathcal{R}[z]$ is closed under addition and multiplication, so both are semirings. The last assertion follows from the fact that $\mathcal{R}_{j} z^{j} \mathcal{R}_{k} z^{k} \subseteq \mathcal{R}_{j+k} z^{j+k}$.

Definition 1.16. Suppose $\mathcal{A}$ is a semialgebra over a commutative base semiring ${ }^{\dagger}$. We write $\operatorname{End}(\mathcal{A})$ for the set of module maps $\mathcal{A} \rightarrow \mathcal{A}$. Given $D \in \operatorname{End}(\mathcal{A})^{S}$, we write $D_{s}$ for the map given by $s \mapsto D(s)$ and $s^{\prime} \mapsto \mathbb{D}, \forall s^{\prime} \neq s$. In the other direction, given $f_{s}: \in \operatorname{End}(\mathcal{A}), s \in S$, each of singleton support $\{s\}$ with the $\{s\}$ distinct, define $D^{f} \in \operatorname{End}(\mathcal{A})^{S}$ via $D^{f}(s)=f_{s}$.

Remark 1.17. $D^{f}(s)(a b)=D^{f}(s)(a) D^{f}(s)(b), \forall a, b \in \mathcal{A}$, under the usual product, seen by matching terms in the left side and the right side. 
1.5. Higher derivations. This discussion applies to any semialgebra $\mathcal{A}$, not necessarily associative and not necessarily a triple. A derivation $\delta: \mathcal{A} \rightarrow \mathcal{A}$ is a map in $\operatorname{End}(\mathcal{A})$ satisfying $\delta(a b)=a \delta(b)+\delta(a) b$. The following concepts were introduced by Hasse and Schmidt [10] and studied further by Heerema [11 13].

For $S$ convolution admissible (not necessarily associative), a homogeneous map $D$ in $(\text { End } \mathcal{A})^{S}$ is called a higher derivation of $A$ if it satisfies the conditions:

(i) $D_{s}(a b)=\sum_{s^{\prime}+s^{\prime \prime}=s} D_{s^{\prime}}(a) D_{s^{\prime \prime}}(b), \quad \forall s \in S, \quad \forall a, b \in \mathcal{A}$.

(ii) $D_{0}=\mathbb{1}$ (the identity map on $A$ ).

Property (i) is called the Leibniz rule, obtained from the more familiar Leibniz rule for derivations for $S=\mathbb{N}$ by dividing by $k$ !.

[10, pp. 190-191] indicates how to define a higher derivation $D$. We have a somewhat different take, along classical lines. We consider semialgebras over semifields containing $\mathbb{Q}_{>0}$, for the following definition to make sense. Given a map $f: \mathcal{A} \rightarrow \mathcal{A}[[z]]$ we define its exponential $\exp (f)=\sum_{j \geq 1} \frac{f^{j}}{j !}: \mathcal{A} \rightarrow \mathcal{A}[[z]]$. It is well-known that the exponential of a derivation is a homomorphism. In fact we have:

Lemma 1.18. If $d_{1}, d_{2}, \ldots$ is a sequence of derivations, then $\sum d_{k} z^{k}: \mathcal{A} \rightarrow \mathcal{A}[[z]]$ satisfies Leibniz' rule. Its exponential is a semialgebra homomorphism: $D:=$ $\sum D_{r} z^{r}:=\exp \left(\sum_{k \geq 1} d_{k} z^{k}\right): \mathcal{A} \rightarrow \mathcal{A}[[z]]$.

Proof. Given in [24] and [4, Propositions 3.4.2 and 3.4.3]. The proof evidently extends to semialgebras over semifields containing $\mathbb{Q}_{>0}$.

Matching coefficients in Lemma 1.18, one gets precisely the Schur polynomials associated to the sequence $d_{1}, d_{2}, \ldots$ In particular:

$D_{1}=d_{1}, \quad D_{2}=\frac{d_{1}^{2}}{2}+d_{2}, \quad D_{3}=\frac{d_{1}^{3}}{3 !}+d_{1} d_{2}+d_{3}, \quad D_{4}=\frac{d_{1}^{4}}{4 !}+\frac{1}{2} d_{1}^{2} d_{2}+\frac{1}{2} d_{2}^{2}+d_{1} d_{3}+d_{4}$, defines a higher derivation $D$.

When each $d_{s}=\delta$ for a given derivation $\delta$, we call $D$ the higher derivation of $\delta$.

\section{Grassmann Semialgebras}

Suppose $\mathcal{A}$ is a commutative semiring and $V$ is an $\mathcal{A}$-module. $\mathfrak{H}$ will denote an $\mathcal{A}$-semialgebra generated by $V$ (Often $\mathfrak{H}$ will be the tensor algebra $T(V)$ defined below). We write $\mathcal{T}_{\mathfrak{H} k}$ for the products of length $k$ of elements of $V$, and $\mathfrak{H}_{\geq k}$ for the ideal $\sum_{j \geq k} \mathcal{T}_{\mathfrak{H} j}$ (with repetitions). Thus $\mathfrak{H}=\mathcal{A}+V+\mathfrak{H}_{\geq 2}$. The elements of $\mathcal{T}_{\mathfrak{H} k}$ and $\mathcal{T}_{\mathfrak{H} l}$ will satisfy $w_{k} w_{l}^{\prime}=(-1)^{k l} w_{l}^{\prime} w_{k}$, leading to the subject of our study.

Definition 2.1. A Grassmann, or exterior, semialgebra, over a semiring ${ }^{\dagger} \mathcal{A}$ and an $\mathcal{A}$-module $V$, is a semialgebra $\mathfrak{H}$ generated by $\mathcal{A}$ and $V$, as above, together with a negation map on $\mathfrak{H}_{\geq 2}$ and an associative wedge product $\wedge: \mathfrak{H} \times \mathfrak{H} \rightarrow \mathfrak{H}$ satisfying

$$
v_{1} \wedge v_{2}=(-) v_{2} \wedge v_{1} \quad \text { for } \quad v_{i} \in V .
$$

Thus $v_{\pi(1)} \wedge \cdots \wedge v_{\pi(t)}=(-)^{\pi} v_{1} \wedge \cdots \wedge v_{t}$ for $t \geq 2$, where $(-)^{\pi}$ denotes the sign of the permutation. This ties in with the theory of triples since, taking $\mathcal{T}(V)$ to be the nonzero products of elements of $V$, then for $k \geq 2,\left(\mathfrak{H}_{\geq 2}, \mathcal{T}(V)_{\geq 2},(-)\right)$ often is a triple, for (-) suitably defined (as in Theorem 2.4). Since $\mathcal{T}(V)_{\geq 2} \subset \mathfrak{H}_{\geq 2}$, it can 
be bypassed by restricting functions, but the negation map (-) will play a crucial role.

Lemma 2.2. If $V$ is spanned by $\left\{b_{i}: i \in I\right\}$, then to verify the Grassmann relation (2.1) it is enough to check that

$$
b_{i} \wedge b_{j}=(-) b_{j} \wedge b_{i}, \quad \forall i, j \in I .
$$

Proof. Distributivity yields

$$
\begin{aligned}
\left(\sum \alpha_{i} b_{i}\right) \wedge\left(\sum \beta_{j} b_{j}\right) & =\sum \alpha_{i} \beta_{j} b_{i} \wedge b_{j}=(-) \sum \alpha_{i} \beta_{j} b_{j} \wedge b_{i} \\
& =(-)\left(\sum \beta_{j} b_{j}\right) \wedge\left(\sum \alpha_{i} b_{i}\right),
\end{aligned}
$$

yielding the assertion.

We write $v^{k}$ for $v \wedge \cdots \wedge v$ taken $k$ times.

Lemma 2.3. $\left(\sum \alpha_{i} a_{i}\right)^{2} \succeq_{\circ} \sum \alpha_{i}^{2} a_{i}^{2}$ for any Grassmann semialgebra.

Proof. $\left(\sum \alpha_{i} a_{i}\right)^{2}=\sum \alpha_{i}^{2} a_{i}^{2}+\sum_{i<j} \alpha_{i} \alpha_{j}\left(a_{i} \wedge a_{j}+a_{j} \wedge a_{i}\right)$.

To obtain Grassmann semialgebras via Definition 2.10 below, we follow the familiar construction of the Grassmann algebra over a module $V$, but with modifications necessitated by working over semirings.

Accordingly, as in [21, Remark 6.35] and [16, Definition 6.10], we define the tensor semialgebra $T(V)=\bigoplus_{n} V^{\otimes(n)}$ (adjoining a copy of $\mathcal{A}$ if we want to have a unit element), with the usual multiplication $v v^{\prime}:=v \otimes v^{\prime}$.

Theorem 2.4. Write $T(V)_{\geq 2}$ for $\bigoplus_{n \geq 2} V^{\otimes(n)}$. Then $T(V)_{\geq 2}$ has a negation map (-) satisfying

for $b_{i_{j}} \in V$.

$$
b_{\pi\left(i_{1}\right)} \otimes \cdots \otimes b_{\pi\left(i_{t}\right)}=(-)^{\pi} b_{i_{1}} \otimes \cdots \otimes b_{i_{t}}
$$

Proof. By Lemma 2.2 we may take a generating set $\left\{b_{i}: i \in I\right\}$ of $V$, where $I$ is an ordered index set. We define a negation on $V \otimes V$ by $(-) b_{i} \otimes b_{j}=b_{j} \otimes b_{i}$. (This is possible since it preserves the bilinear relations defining the tensor product.) Since this is homogeneous of degree 2 , it defines a negation on $\mathfrak{G}(V)_{2}$ given by $(-) b_{i} \otimes b_{j}=$ $b_{j} \otimes b_{i}$. When $i<j$ we thus rename $b_{j} \otimes b_{i}$ as $(-) b_{i} \otimes b_{j}$. It is easy to see that this is the same as defining a reduction procedure. Thus $b_{\pi\left(i_{1}\right)} \cdots b_{\pi\left(i_{t}\right)} \mapsto(-)^{\pi} b_{i_{1}} \cdots b_{i_{t}}$, where $\pi$ is the permutation rearranging the indices $i_{1} \ldots, i_{t}$ in ascending order. We get $(-)^{\pi}$ by writing $\pi$ as a product of transpositions; since $(-)^{\pi}$ is independent of the way we write $\pi$ in this manner, our reduction procedure is well-defined, cf. [20].

We continue to develop the Grassmann theory. We can eliminate many occurrences of $(-)$ in our formulas by switching two of the $b_{i}$. The tricky part is dealing with degree 1, i.e., in $V$ itself, where we cannot perform this switch. But issues like determinants and linear independence of $n$ vectors are trivial for $n=1$, thereby enabling us to forego (-) on elements of degree 1 . In this manner, our way out in $\$ 3$ is to focus on elements of degree $>1$.

Definition 2.5. $\mathcal{T}_{\text {even }}^{\geq 2}$ is the set of all even products of elements of $V$, not including the constants $\mathcal{A}, \mathfrak{G}$ even $\geq 2$ is the submodule of $\mathfrak{G}$ generated by $\mathcal{T}_{\text {even }}^{\geq 2}, \mathcal{T}_{\text {odd }}$ is the set of all odd products of elements of $V$, and $\mathfrak{G}_{\text {odd }}$ is the submodule of $\mathfrak{G}$ generated by $\mathcal{T}_{\text {odd }}$. 
Lemma 2.6. If $v \in \mathfrak{G}_{i}$ and $v^{\prime} \in \mathfrak{G}_{j}$ for $i, j \geq 1$ then

$$
v \wedge v^{\prime}=(-)^{i+j} v^{\prime} \wedge v,
$$

where (-) is given as in Theorem 2.4.

Proof. Easy induction on $i$ and $j$.

Definition 2.7. $T(V)^{\circ \sharp}$ is the ideal of $T(V)$ generated by $T(V)^{\circ}$ and all elements $v \otimes v, v \in V$.

(This is just $T(V)^{\circ}$ when $\frac{1}{2} \in \mathcal{A}$ since then $\left.v \otimes v=\left(\frac{1}{2} v \otimes v\right)^{\circ}\right)$. We now weaken $\preceq$.

Definition 2.8. (Supplanting Definition [1.6) $a_{0} \preceq a_{1}$ in $T(V)$ if $a_{1}=a_{0}+d$ for some $d \in T(V)^{\circ \sharp}$.

2.0.1. The standard Grassmann semialgebra. Recall that the way to define factor structures in universal algebra (in particular, for semirings ${ }^{\dagger}$ or modules over semirings ${ }^{\dagger}$ ) is to mod out by a congruence.

Theorem 2.9. If $v^{2}=\mathbb{O}$ for all $v$ in $V$, then for any permutation $\pi$ and all $v_{i} \in V$,

(i) $v \wedge v_{1} \wedge \cdots \wedge v_{n} \wedge v=\mathbb{0}$.

(ii) $v_{\pi(1)} \wedge \cdots \wedge v_{\pi(n)}=v_{1} \wedge \cdots \wedge v_{n}$ if $\pi$ is even;

(iii) $v_{1} \wedge \cdots \wedge v_{n}+v_{\pi(1)} \wedge \cdots \wedge v_{\pi(n)}=\mathbb{0}$ if $\pi$ is odd.

Thus the only quasi-zeros are 0.

Proof. Linearizing yields

$$
\mathbb{D}=\left(v_{1}+v_{2}\right)^{2}=v_{1}^{2}+v_{2}^{2}+v_{1} \wedge v_{2}+v_{2} \wedge v_{1}=\mathbb{O}+\mathbb{O}+v_{1} \wedge v_{2}+v_{2} \wedge v_{1},
$$

so $v_{1} \wedge v_{2}+v_{2} \wedge v_{1}=\mathbb{0}$. Now (i) is by induction on $n$, since $v \wedge v_{1} \wedge \cdots \wedge v_{n} \wedge v=$ $v \wedge v_{1} \wedge \cdots \wedge v_{n} \wedge+\mathbb{O}=\left(v \wedge v_{1}+v_{1} \wedge v\right) \wedge \cdots \wedge v_{n} \wedge v=\mathbb{O}$.

To get (ii) and (iii) we write $\pi$ as a product of transpositions $\pi_{1} \cdots \pi_{k}$ of the form $(i, i+1)$. If $k=2$ then $v_{1} \wedge v_{2} \wedge v_{3}=v_{1} \wedge v_{2} \wedge v_{3}+\left(v_{2} \wedge\left(v_{1} \wedge v_{3}+v_{3} \wedge v_{1}\right)\right)=$ $\left(v_{1} \wedge v_{2}+v_{2} \wedge v_{1}\right) \wedge v_{3}+v_{2} \wedge v_{3} \wedge v_{1}=v_{2} \wedge v_{3} \wedge v_{1}$, and then we have (ii) for all even $k$.

For $k$ odd, we use (ii) to reduce to $v_{1} \wedge v_{2} \wedge \cdots \wedge v_{n}+v_{2} \wedge v_{1} \wedge \cdots \wedge v_{n}=\mathbb{0}$.

The last assertion follows by using these equalities to reduce every quasi-zero until reaching $\mathbb{D}$.

Definition 2.10. The standard Grassmann semialgebra $\wedge V$ with respect to a given generating set $\left\{b_{i}: i \in I\right\}$ of $V$, also denoted $\mathfrak{G}(V)$, is $T(V) / \Phi$, where $\Phi$ is the congruence generated by $(v \wedge v, \mathbb{O}), \forall v \in V$. Accordingly $\mathfrak{G}(V)_{k}$ is $T(V)_{k} / \Phi$ and $\mathfrak{G}(V)_{\geq 2}=T(V)_{\geq 2} / \Phi$.

The standard Grassmann triple is $\left(\mathfrak{G}(V)_{\geq 2}, \mathcal{T}_{\mathfrak{G}(V)_{\geq 2}},(-)\right)$, where $\mathcal{T}_{\mathfrak{G}(V)_{\geq 2}}$ is the product of elements of $V$ of length $\geq 2$, and $(-)$ is as in Theorem 2.4

2.1. Symmetrization and the twist action. There is a general way to provide a negation map for arbitrary Grassmann semialgebras. Although $\mathcal{T}$-modules initially may lack negation, one can obtain negation maps for them through the next main idea, the symmetrization process, which although a special case of supersemialgebras and their modules, provides a crucial method of creating a triple. Tropical symmetrization dates back to [7, and we recall the treatment for systems from [16]. 
Definition 2.11. Given any $\mathcal{T}$-monoid module $\mathcal{M}$, define its $\mathbb{Z}_{2}$-graded symmetrization $\widehat{\mathcal{M}}=\mathcal{M} \times \mathcal{M}$, with componentwise addition.

Also define $\widehat{\mathcal{T}}=(\mathcal{T} \times\{\mathbb{O}\}) \cup(\{\mathbb{O}\} \times \mathcal{T})$ with the twist action of $\widehat{\mathcal{T}}$ on $\widehat{\mathcal{M}}$ given by the super-action, namely

$$
\left(a_{0}, a_{1}\right) \cdot_{\text {tw }}\left(c_{0}, c_{1}\right)=\left(a_{0} c_{0}+a_{1} c_{1}, a_{0} c_{1}+a_{1} c_{0}\right), \quad a_{i} \in \mathcal{T}, c_{i} \in \mathcal{M}
$$

Definition 2.12. The switch map $(-)_{\text {sw }}$ on the symmetrized module $\widehat{\mathcal{M}}$ is given by $(-)_{\mathrm{sw}}\left(c_{0}, c_{1}\right)=\left(c_{1}, c_{0}\right)$.

If $\mathcal{A}$ is a semiring containing $\mathcal{T}$, then we define the twist action as in (2.3), but this time with $a_{i}, c_{i} \in \mathcal{A}$.

Theorem 2.13 ([15, Theorems 2.41, 2.43]). For any $\mathcal{T}$-module $\mathcal{A}$, we can embed $\mathcal{A}$ into $\widehat{\mathcal{A}}$ via

$$
b \mapsto(b, \mathbb{O}),
$$

thereby obtaining a faithful functor from the category of semirings into the category of semirings with a negation map (and preserving additive idempotence). This also yields a faithful functor from ordered semigroups to signed (-) $)_{\mathrm{sw}}$-bipotent systems. Any $\mathcal{A}$-module $\mathcal{M}$ yields a $\widehat{\mathcal{A}}$-module $\widehat{\mathcal{M}}=\mathcal{M} \oplus \mathcal{M}$, which has a signed decomposition where $\mathcal{M}^{+}$is the first component.

This applies to the Grassmann semialgebra:

Theorem 2.14. Define $\mathfrak{G}(V)^{\natural}$ to be $\widehat{\mathfrak{G}(V)}$ modded out by the congruence generated by $\left(v \wedge v^{\prime}, 0\right) \cong\left(0, v^{\prime} \wedge v\right)$ for all $v, v^{\prime} \in V$, and $\overline{\mathcal{T}}$ to be the corresponding image of $\widehat{\mathcal{T}}$. There is a triple $\left(\mathfrak{G}(V)^{\natural}, \widehat{\mathcal{T}},(-)_{\mathrm{sw}}\right)$, together with an embedding of triples

$$
\left(\mathfrak{G}(V)_{\geq 2}, \mathcal{T}_{\mathfrak{G}(V)_{\geq 2}},(-)\right) \rightarrow\left(\mathfrak{G}(V)^{\natural}, \overline{\mathcal{T}},(-)_{\text {sw }}\right)
$$

given by $c \mapsto(c, 0)$.

Proof. Take $\mathcal{A}=\mathfrak{G}(V)$ in Theorem 2.13 ,

$$
(-)\left(v \wedge v^{\prime}\right)=v^{\prime} \wedge v \mapsto\left(v^{\prime} \wedge v, 0\right)=(-)_{\mathrm{sw}}\left(0, v^{\prime} \wedge v\right)=(-)_{\mathrm{sw}}\left(v \wedge v^{\prime}, 0\right) .
$$

Thus (-) matches by (2.13), so the Grassmann relations match.

2.2. The partially reduced Grassmann system (when $V$ is free). The main results of this paper involve the free module $V$ with base $\mathcal{B}=\left\{b_{0}, \ldots, b_{n-1}\right\}$, in the sense that any element of $V$ can be written uniquely as an $\mathcal{A}$-linear combination of the $b_{i}$. Let $V_{n}:=\mathcal{A}^{(n)}$ be the free module over the semiring $\mathcal{A}$ with basis $\mathbf{b}:=\left\{b_{0}, \ldots, b_{n-1}\right\}$ of $n$ elements. When $V=V_{n}$, this includes the definition in [8. Definition 3.1.2], in which $(-)$ is the identity map. In this work we have two candidates for the Grassmann algebra, given respectively in Theorems 2.14 and 2.22 .

Remark 2.15.

(i) By Theorem 2.9, $b_{\pi\left(i_{1}\right)} \wedge \cdots \wedge b_{\pi\left(i_{t}\right)}=(-)^{\pi} b_{i_{1}} \wedge \cdots \wedge b_{i_{t}}$ for any permutation $\pi$. Also, every simple tensor in which some $b_{i}$ repeats is $\mathbb{O}$.

(ii) $\mathfrak{G}_{k}$ is free with a base of $2\left(\begin{array}{c}n \\ k\end{array}\right)$ elements. For instance $\mathfrak{G}_{2}\left(V_{3}\right)$ has base $b_{1} \wedge b_{2}, b_{1} \wedge b_{3}, b_{2} \wedge b_{3}$ and their "negations" $b_{2} \wedge b_{1}, b_{3} \wedge b_{1}, b_{3} \wedge b_{2}$. This phenomenon gives rise to the "eigenvalue pair" of $\$ 3.2$ below. 
Lemma 2.16. For the free Grassmann semialgebra, $\mathfrak{G}=\mathfrak{G}_{\text {even }} \oplus \mathfrak{G}_{\text {odd }}$ is a supersemialgebra, and its ideal $\mathfrak{G}^{\geq 2}=\mathfrak{G}_{\text {even }}^{\geq 2} \oplus \mathfrak{G}_{\text {odd }}^{\geq 2}$ has the negation map from Theorem 2.4

Proof. By linearity, we need only check products of the $b_{i}$.

Lemma 2.17. (-) is well-defined, and

$$
\bar{b}_{\pi\left(i_{1}\right)} \wedge \cdots \wedge \bar{b}_{\pi\left(i_{t}\right)}=(-)^{\pi} \bar{b}_{i_{1}} \wedge \cdots \wedge \bar{b}_{i_{t}}, \quad \forall t \geq 2 .
$$

Proof. (-) is well-defined by Theorem 2.4. The formula follows from writing a permutation as the product of transpositions, noting that the sign of a permutation is well-defined, and counting the number of times $(-)$ occurs.

Lemma 2.18. Suppose $\sum_{\mathbf{i}} \alpha_{\mathbf{i}} b_{i_{1}} \otimes \cdots \otimes b_{i_{k}}+d \preceq \sum_{\mathbf{i}} \alpha_{\mathbf{i}^{\prime}} b_{i_{1}}^{\prime} \otimes \cdots \otimes b_{i_{k}}^{\prime}+d^{\prime}$, where $i_{1}<\cdots<i_{k}, \alpha_{\mathbf{i}}, \alpha_{\mathbf{i}^{\prime}} \in \mathcal{A}, d, d^{\prime} \in \overline{\mathfrak{G}^{\circ}}$. Then $\sum_{\mathbf{i}} \alpha_{\mathbf{i}} b_{i_{1}} \otimes \cdots \otimes b_{i_{k}} \preceq \circ \sum_{\mathbf{i}} \alpha_{\mathbf{i}^{\prime}} b_{i_{1}}^{\prime} \otimes \cdots \otimes b_{i_{k}}^{\prime}$, where $i_{1}<\cdots<i_{k}$.

Proof. Match components, eliminating those components in which some $b_{i}$ repeats or some of the $b_{i}$ descend.

Definition 2.19. $T(V)_{\text {doub }}$ is the ideal of $T(V)$ generated by all elements $b_{i} \otimes b_{i}$ for all $i$.

Lemma 2.20. Any nonzero element of $T(V)$ is a sum of terms $( \pm) \alpha b_{i_{1}} \cdots \otimes b_{i_{k}}+d$, where $i_{1}<\cdots<i_{k}, \alpha \in \mathcal{A}$, and $d \in T(V)_{\text {doub }}$.

Proof. We rearrange the $b_{i}$ appearing in the summands, noting that any time a $b_{i}$ repeats, the product is in $T(V)_{\text {doub }}$.

We use Lemma 2.18 to avoid $T(V)_{\text {doub }}$ in our computations. We will need the following nondegeneracy result.

Proposition 2.21. Suppose $V=\mathcal{A}^{(n)}$ and $u, u^{\prime} \in \mathfrak{G}(V)_{k}$ for $2 \leq k<n$.

(i) If $u \wedge v=u^{\prime} \wedge v$ for all $v \in \mathfrak{G}(V)_{n-k}$, then $u=u^{\prime}$.

(ii) If $u \notin T(V)_{k}^{\circ}$ then there is some $v \in T(V)_{n-k}$ for which $u \wedge v \notin T(V)_{k}^{\circ}$.

Proof. Using Lemma 2.20, write

$$
u=\sum_{i_{1}<\cdots<i_{k}} \alpha_{i_{1}, \ldots i_{k}} b_{i_{1}} \wedge \cdots \wedge b_{i_{k}}, u^{\prime}=\sum_{i_{1}<\ldots i_{k}} \alpha_{i_{1}, \ldots i_{k}}^{\prime} b_{i_{1}^{\prime}} \wedge \cdots \wedge b_{i_{k}^{\prime}} .
$$

(i) For any $\alpha_{1, \ldots k} \neq \mathbb{O}, u \wedge b_{i_{k+1}} \wedge \cdots \wedge b_{i_{n}}=( \pm) \alpha_{1, \ldots k} b_{1} \wedge \cdots \wedge b_{n}$, which must be $( \pm) \alpha_{1, \ldots k}^{\prime} b_{1} \wedge \cdots \wedge b_{n}$, with the base elements matching up.

(ii) Adjusting notation, we may assume that $\alpha_{1, \ldots k} \neq 0$. But then

$$
u \wedge b_{k+1} \wedge \cdots \wedge b_{n}=\alpha_{1, \ldots k} b_{1} \wedge \cdots \wedge b_{n} \notin T(V)_{n}^{\circ \sharp} .
$$

Theorem 2.22. More in line with [8], define the partially reduced Grassmann algebra $\mathfrak{G}(V)_{\geq 2}^{\diamond}$ to be $T(V)_{\geq 2}$ modded out by the congruence generated by $\left(b_{i} \wedge b_{i}, 0\right)$ for all $i, \mathfrak{G}(V)^{\sharp}$ to be $\widehat{T(V)}$ modded out by the congruence generated by $\left(b_{i} \wedge b_{j}, 0\right) \cong$ $\left(0, b_{j} \wedge b_{i}\right)$ for all $i, j$, and $\widehat{\mathcal{T}}^{\sharp}$ to be the corresponding image of $\widehat{\mathcal{T}}$.

There is a triple $\left(\mathfrak{G}(V)^{\sharp}, \widehat{\mathcal{T}}^{\sharp},(-)_{\mathrm{sw}}\right)$, together with an embedding of triples

$$
\left(\mathfrak{G}(V)_{\geq 2}^{\diamond}, \mathcal{T}_{\mathfrak{G}(V)_{\geq 2} \diamond},(-)\right) \rightarrow\left(\mathfrak{G}(V)^{\sharp}, \mathcal{T}^{\sharp},(-)_{\mathrm{sw}}\right)
$$

given by $c \mapsto(c, 0)$. 
Note 2.23. $\mathfrak{G}(V)_{\geq 2}$ is clearly a homomorphic image of $\mathfrak{G}(V)_{\geq 2}^{\diamond}$, since it has more relations. (All $v \otimes v$ are sent to $\mathbb{O}$, not just the $b_{i} \otimes b_{i}$.) Although $\mathfrak{G}(V)_{\geq 2}^{\diamond}$ and $\mathfrak{G}(V)_{\geq 2}$ both coincide with the Grassmann algebra in the classical setting where $V$ is a vector space over a field, they differ in the semiring setting, since $b_{i} \otimes b_{j}+b_{j} \otimes b_{i}$ is not $\mathbb{O}$ in $\mathfrak{G}(V)_{\geq 2}^{\#}$.

2.3. Digression: Related notions. For the remainder of this section we examine algebraic notions related to this paper, even though one can bypass them for the proofs of Theorems 3.14 and 3.15 .

2.3.1. The case when $V$ already has a negation map. We have seen that $V$ itself need not have a negation map, for us "almost" to define a negation map on $T(V)$. In case $V$ does have a negation map $(-) 2$ we need a slight modification. We define a negation map on the tensor product $V \otimes W$ by $(-)(v \otimes w)=((-) v) \otimes w$. When $W$ also has a negation map $(-)$ we define a negated tensor product $V \otimes_{(-)} W$ by imposing the extra axiom

$$
((-) v) \otimes_{(-)} w=v \otimes_{(-)}((-) w), \quad v \in V, w \in W .
$$

(One mods out the tensor product by the congruence generated by all elements $((-) v \otimes w, v \otimes(-) w)$.

Remark 2.24. The appropriate triple is $\left(\mathfrak{G}, \mathcal{T}_{\mathfrak{G}},(-)\right)$, where $\mathcal{T}_{\mathfrak{G}}=\left\{v_{1} \wedge \cdots \wedge v_{t}\right.$ : $\left.v_{i} \in V, t \in \mathbb{N}\right\}$, the submonoid generated by $\mathcal{T}$, with $(-)\left(v_{1} \wedge \cdots \wedge v_{t}\right)=$ $\left((-) v_{1}\right) \wedge \cdots \wedge v_{t}$.

2.3.2. Comparison with [8]. The arguments of [8, whose objective is to obtain a Grassmann algebra point of view for Plücker relations, can be adapted to this situation. We use the systemic version which enables us to replace the "bend relation" $f \sim g$ of [8] for $f, g \in \operatorname{Hom}(V, \mathcal{A})$ by $f+g$ being a quasi-zero in the sense that $(f+g)(v) \in \mathcal{A}^{\circ}$ for every $v \in V$. Then the Plücker relations in 8 , Proposition 4.1.2] become the conditions that $\sum_{i \in A \backslash B} v_{A-\{i\}} v_{B+\{i\}}$ is a quasi-zero.

Note 2.25. The flavor of the Grassmann algebra might be better preserved by taking the negation map (-) not to be the identity map, but rather as defined here, which also could be obtained using symmetrization. Note also that $\mathfrak{G}$ is commutative in [8, Definition 3.1.2]. So why does [8, Definition 3.1.2] work? The answer is that $\mathfrak{G}$ is largely a book-keeping devise to keep track of sets of vectors without repetition, and application of its theory to matroids does not require much of multiplication other than $e_{i} \wedge e_{i}=0$. [8, Proposition 3.1.4] is formal. One needs a cancelation result parallel to [8, Lemma 3.2.2], and [8, Proposition 4.2.1] requires the ability to switch vectors $e_{i}$ and $e_{j}$.

Remark 2.26. Suppose that $\mathcal{A}$ is "zero sum free" in the sense that $a_{1}+a_{2}=\mathbb{O}$ implies $a_{1}=a_{2}=\mathbb{O}$. Then the base $\mathcal{B}$ of a free module $V$ is unique up to multiplication of invertible elements of $\mathcal{A}$. (Otherwise some $b_{i}$ does not appear in the new base, and we cannot recover $b_{i}$ since we cannot zero out extraneous coefficients.)

\footnotetext{
${ }^{2}$ For example $V$ could be the free $\mathcal{A}$-module with a negation map, with base $\left\{b_{i},(-) b_{i}: i \in I\right\}$.
} 


\subsubsection{Digression: The Grassmann envelope.}

Remark 2.27. Just as with classical algebra, one can use $\mathfrak{G}$ to study a supersemialgebra $\mathcal{A}=\mathcal{A}_{0} \oplus \mathcal{A}_{1}$ using its Grassmann envelope $\mathcal{A}_{0} \otimes \mathfrak{G}_{0}+\mathcal{A}_{1} \otimes \mathfrak{G}_{1}$ $\subset \mathcal{A} \otimes \mathfrak{G}$. Following Zelmanov, we say that a super-semialgebra $\mathcal{A}$ is super-P if its Grassmann envelope is $\mathrm{P}$. For example, $\mathcal{A}$ is super-commutative if its Grassmann envelope is commutative. In particular, $\mathfrak{G}$ itself is super-commutative.

Then one can study linear algebra over super-commutative super-semialgebras, super-anticommutative super-semialgebras, and so forth, as indicated in [21, §8.2.2].

\section{Hasse-Schmidt DeRivations on Grassmann SEMi-Algebras}

Having set out the general framework, let us turn to the situation at hand. We review our set-up, in the special case of power series over endomorphisms of the Grassmann algebra. As before, $V_{n}:=\mathcal{A}^{(n)}$ is the free module with basis $\mathbf{b}:=$ $\left\{b_{0}, \ldots, b_{n-1}\right\}$. (We start our subscripts with 0 in consonance with the notation for projective space.) Let $T_{0}\left(V_{n}\right)=\mathcal{A}$, and $T_{k}\left(V_{n}\right):=V_{n} \otimes V_{n} \otimes \cdots \otimes V_{n}$ be its $k$ tensor power. Define a negation $(-): T_{2}\left(V_{n}\right) \rightarrow T_{2}\left(V_{n}\right)$ by mapping $u \otimes v$ to $v \otimes u$. In particular $(-)(u \otimes u)=u \otimes u$. We extend this to $(-): T_{k}\left(V_{n}\right) \rightarrow T_{k}\left(V_{n}\right)$ by means of Theorem 2.4 and Lemma 2.17 Let

$$
T_{\geq 2}\left(V_{n}\right)=\{\mathbb{0}\} \cup \bigoplus_{k \geq 2} T_{k}\left(V_{n}\right),
$$

a semiring ${ }^{\dagger \dagger}$ with multiplication given by tensoring. We consider two variants of the Grassmann algebra:

(1) $\mathfrak{G}(V)_{\geq 2}^{\sharp}$, modding out by the congruence $\mathcal{I}$ of $T_{\geq 2}\left(V_{n}\right)$ generated by all $\left\{\left(b_{i} \otimes b_{i}, 0\right): 0 \leq i<n\right\}$,

(2) $\mathfrak{G}(V)_{\geq 2}$, modding out by the congruence $\mathcal{I}$ of $T_{\geq 2}\left(V_{n}\right)$ generated by all $\left\{(u \otimes u, 0): u \in V_{n}\right\}$.

In either case we will work with a graded Grassmann semialgebra $\mathfrak{G}$, which now we denote as

$$
\bigwedge V_{n}=\bigoplus_{r \geq 0} \bigwedge^{r} V_{n}, \quad \text { where }
$$

$$
\bigwedge^{0} V_{n}=\mathcal{A}, \quad \bigwedge^{1} V_{n}=V_{n}, \quad \text { and } \quad \bigwedge^{r} V_{n}:=\frac{T_{r}\left(V_{n}\right)}{\mathcal{I} \cap T_{r}\left(V_{n}\right)} \quad \text { for } r \geq 2 .
$$

Thus $u \wedge v$ denotes the image of $u \otimes v$ through the natural map $T\left(V_{n}\right) \rightarrow \bigwedge V_{n}$. Here $\preceq$ is $\preceq$ 。 .

Remark 3.1. By Theorem 2.4, each submodule $\bigwedge^{r} V_{n}, r \geq 2$, inherits a negation map by putting

$$
(-)\left(u_{1} \wedge u_{2} \cdots \wedge u_{r}\right)=u_{2} \wedge u_{1} \wedge \cdots \wedge u_{r}
$$

Remark 3.2.

(i) For each $r \geq 2, \wedge^{r} V_{n}$ is spanned by words $b_{i_{0}} \wedge b_{i_{1}} \wedge \cdots \wedge b_{i_{r-1}}$ of length $r$. In particular, $\bigwedge^{r} V_{n}$ is a free $\mathcal{A}$ module spanned by $[\mathbf{b}]_{\boldsymbol{\lambda}}^{r}$, where

$$
\boldsymbol{\lambda}:=\left(\lambda_{1} \geq \cdots \geq \lambda_{r}\right), \quad[\mathbf{b}]_{\lambda}^{r}:=b_{\lambda_{r}} \wedge b_{1+\lambda_{r-1}} \wedge \cdots \wedge b_{r-1+\lambda_{1}}
$$


We are interested in the $\mathbb{N}$-graded power series semiring $\left(\bigwedge V_{n}\right)[[z]]:=\oplus_{r \geq 0} \bigwedge V_{n} z^{r}$ of Definition 1.14 (and later its super-version), and its endomorphisms.

Since the congruences are homogeneous, we define

$$
\bigwedge^{\geq 1} V_{n}:=\bigoplus_{r \geq 1} \bigwedge^{r} V_{n}, \quad \bigwedge^{\geq 2} V_{n}:=\bigoplus_{r \geq 2} \bigwedge^{r} V_{n}, \quad \text { and } \quad \bigwedge^{\neq 1} V_{n}:=\bigoplus_{r \neq 1} \bigwedge^{r} V_{n}
$$

Definition 3.3. Let $D\{z\}:=\sum_{i \geq 0} D_{i} z^{i} \in \operatorname{End}\left(\bigwedge V_{n}\right)[[z]]$ be homogeneous of degree 0 (i.e. $D_{i}\left(\bigwedge^{r} V_{n}\right) \subseteq \bigwedge^{r} V_{n}$ and in particular $\left.\left.D_{i}\left(V_{n}\right) \subseteq V_{n}\right)\right)$. If

$$
D\{z\}(u \wedge v)=D\{z\} u \wedge D\{z\} v
$$

we say that it is a Hasse-Schmidt (HS) derivation on $\bigwedge V_{n}$.

To simplify notation let us simply denote the identity map on $V_{n}$ as " $\mathbb{1}_{V}$," also identified with $D\{z\}$ where $D_{0}=\mathbb{1}$ and all other $D_{i}=\mathbb{0}$.

Equation (3.2) is equivalent to:

$$
D_{k}(u \wedge v)=\sum_{i+j=k} D_{i} u \wedge D_{j} v, \quad \forall k \geq 0, \quad \forall u, v \in \bigwedge V_{n} .
$$

For $r \geq 2$, any element of $\bigwedge^{r} V_{n}$ is a linear combination of monomials $v_{1} \wedge \cdots \wedge v_{r}$ of length $r$. The definition shows that $D\{z\}$ is uniquely determined by the values it takes on elements of $V$.

In the following we shall restrict to a special class of $H S$ derivations, useful for the applications.

Proposition 3.4. For any $f \in \operatorname{End}_{\mathcal{A}}\left(V_{n}\right)$, there exists a unique $H S$-derivation $D^{f}\{z\}$ on $\bigwedge V_{n}$ such that $D^{f}\{z\}_{\left.\right|_{n}}=\sum_{i \geq 0} f^{i} z^{i}$.

Proof. For the chosen $\mathcal{A}$-basis of the module $V$ we necessarily have $D^{f}\{z\}\left(b_{j}\right)=$ $\sum_{i \geq 0} f^{i}\left(b_{j}\right) z^{i}$. Write $f(z)$ for $\sum_{i \geq 0} f^{i} z^{i}$. One defines $D^{f}\{z\}$ on $\bigwedge V$ by setting for each degree:

$$
D^{f}\{z\}\left(b_{i_{1}} \wedge \cdots \wedge b_{i_{j}}\right)=f(z) b_{i_{1}} \wedge \cdots \wedge f(z) b_{i_{j}}, \quad 1 \leq j \leq r .
$$

If $D$ were another derivation satisfying the same initial condition, it would coincide on all the basis elements of $\bigwedge V_{n}$, which generate all elements of $\bigwedge V_{n}$.

Example 3.5. Let us compute $D_{2}^{f}\left(b_{1} \wedge b_{2}\right)$ where $f\left(b_{i}\right)=b_{i+1}$. Then

$$
\begin{aligned}
D_{2}^{f}\left(b_{1} \wedge b_{2}\right) & =D_{2}^{f}\left(b_{1}\right) \wedge b_{2}+D_{1}^{f} b_{1} \wedge D_{1}^{f} b_{2}+b_{1} \wedge D_{2}^{f} b_{2} \\
& =f^{2}\left(b_{1}\right) \wedge b_{2}+f\left(b_{1}\right) \wedge f\left(b_{2}\right)+b_{1} \wedge f^{2}\left(b_{2}\right) \\
& =b_{3} \wedge b_{2}+b_{2} \wedge b_{3}+b_{1} \wedge b_{4} \succeq b_{1} \wedge b_{4},
\end{aligned}
$$

since $b_{3} \wedge b_{2}+b_{2} \wedge b_{3}$ is a quasi-zero.

From now on we shall fix the endomorphism $f$ once and for all, and write $D\{z\}:=$ $D^{f}(z)$ and $D:=D_{1 \mid V}:=f$. Also we write $D_{i} v$ for $D_{i}(v)$ and $D\{z\} v$ for $\sum D_{i} v \cdot z^{i}$. In particular, for each $v \in V_{n}$ the equality $D_{i} v=D_{1}^{i} v=f^{i}(v)$ holds.

Lemma 3.6. For $u, v \in V_{n}$,

(i) $D\{z\} v=v+D\{z\}\left(D_{1} v\right) z$.

(ii) $D\{z\}(u \wedge v)=u \wedge D\{z\} v+z D\{z\}\left(D_{1} u \wedge v\right)$. 
Proof.

(i) $D\{z\} v=v+\sum_{i \geq 1} D_{i} v z^{i}=v+\sum_{i \geq 1}\left(D_{i-1} D_{1} v z^{i-1}\right) z=v+D\{z\}\left(D_{1} v\right) z$.

(ii) $\left.D\{z\}(u \wedge v)=D\{z\} u \wedge D\{z\} v=\left(u+z D\{z\} D_{1} u\right) \wedge D\{z\} v\right)$ $=u \wedge D\{z\} v+z D\{z\} D_{1} u \wedge D\{z\} v=u \wedge D\{z\} v+z D\{z\}\left(D_{1} u \wedge v\right)$.

\subsection{The canonical quasi-inverse of $D\{z\}$.}

Definition 3.7. $\bar{D}\{z\}:=\sum_{i \geq 0} \bar{D}_{i} z^{i} \in \operatorname{End}\left(\bigwedge^{\neq 1} V_{n}\right)[[z]]$ is a (left) quasi-inverse of $D\{z\}$ if

$$
\bar{D}\{z\} D\{z\} u \succeq u, \quad \forall u \in \bigwedge^{\neq 1} V_{n}
$$

Our next task consists in constructing a quasi-inverse $\bar{D}\{z\}$ of the $H S$ derivation $D\{z\}$, that we will achieve through a number of steps necessary to cope with the difficulty of not having a natural negation map on $V_{n}$. This can be done in two ways: First do it in the classical case, and then apply the "transfer principle" of Remark 1.7. However, one gets more precise information by taking the direct analog.

Construction. Towards this purpose we first consider the map:

$$
\left\{\begin{array}{cccc}
\bar{D}: V_{n} & \longrightarrow & \operatorname{End}_{\mathcal{A}}\left(\bigwedge V_{n}\right) \\
u & \longmapsto & \bar{D} u
\end{array}\right.
$$

such that $\bar{D} u(v):=v \wedge D_{1} u$ for all $v \in V_{n}$. If $v \in \bigwedge^{\geq 2} V_{n}$, we may assume it is of the form $v=v_{1} \wedge v_{2}$ with $v_{1} \in V_{n}$. In this case we define

$$
\bar{D} u(v)=\bar{D} u\left(v_{1} \wedge v_{2}\right)=\bar{D} u\left(v_{1}\right) \wedge v_{2}=v_{1} \wedge D_{1} u \wedge v_{2} .
$$

It is easily seen that (3.7) suffices to define $\bar{D} u$ on all the Grassmann semi-algebras and also that the definition does not depend on the representation of the same element $v \in \bigwedge V_{n}$. In fact any one such is a finite linear combination of tensors of the form $v:=v_{1} \wedge \cdots \wedge v_{k}$, and the definition of $\bar{D} u(v)$ does not change if we replace the given expression of $v$ with an equivalent one after an even permutation of the factors. It will be useful to identify $V_{n}$ as a subset of $\operatorname{End}_{\mathcal{A}}\left(\bigwedge V_{n}\right)$, by viewing each of its elements as a (wedge) multiplication operator, under the map $v \mapsto v \wedge$, i.e. $v(w)=v \wedge w$ for all $w \in \wedge V_{n}$.

Definition 3.8. Let $\bar{D}\{z\}:=1+\bar{D}_{1} z+\bar{D}_{2} z^{2}+\cdots+\bar{D}_{n} z^{n}: V_{n} \rightarrow \operatorname{End}_{\mathcal{A}}\left(\bigwedge V_{n}\right)[z]$ defined as follows. If $u_{1} \wedge \cdots \wedge u_{k} \in \wedge^{k} V_{n}$, then

$$
\bar{D}\{z\}\left(u_{1} \wedge \cdots \wedge u_{k}\right)=\bar{D}\{z\}\left(u_{1}\right) \circ \cdots \circ \bar{D}\{z\}\left(u_{k}\right)
$$

where $\circ$ is the composition in $\operatorname{End}_{\mathcal{A}}\left(\bigwedge V_{n}\right)$ and where for all $u \in V_{n}=\bigwedge^{1} V_{n}$ we set

$$
(\bar{D}\{z\} u)(v)=u \wedge v+z \bar{D} u(v) \in \operatorname{End}_{\mathcal{A}}\left(\bigwedge V_{n}\right)[z]
$$

acting on $v \in \bigwedge V_{n}$ as $\bar{D}\{z\} u(v)=u \wedge v+(\bar{D} u) v$. Now we extend $\bar{D}\{z\}$ to a map $\bigwedge V_{n} \rightarrow \operatorname{End}_{\mathcal{A}}\left(\bigwedge V_{n}\right)$ defining it on monomials of degree $k \geq 2$ via $\left(1+\bar{D}_{1} z+\cdots+\bar{D}_{k} z^{k}\right)\left(u_{1} \wedge \cdots \wedge u_{k}\right)=\bar{D}\{z\}\left(u_{1} \wedge \cdots \wedge u_{k}\right)=\bar{D}\{z\}\left(u_{1}\right) \circ \cdots \circ \bar{D}\{z\}\left(u_{k}\right)$

Remark 3.9. By definition it follows that if $u \in \bigwedge^{i} V_{n}$, then $\bar{D}_{j} u=0$ for all $j>i$. 
Example 3.10. In this example we compute $\bar{D}_{1}\left(u_{1} \wedge u_{2}\right), \bar{D}_{2}\left(u_{1} \wedge u_{2}\right)$ and $\bar{D}_{2}\left(u_{1} \wedge u_{2} \wedge u_{3}\right)$ to illustrate how the definition works. By definition $\bar{D}_{1}\left(u_{1} \wedge u_{2}\right)$ and $\bar{D}_{2}\left(u_{1} \wedge u_{2}\right)$ are the coefficients of $z$ and $z^{2}$ in the expansion $\left(\bar{D} u_{1}\right) \circ\left(\bar{D}_{1} u_{2}\right)$ that we apply to a test element $w \in \bigwedge V_{n}$. By definition one may assume $w \in V_{n}$. One has:

$$
\begin{aligned}
& \left(u_{1} \wedge u_{2}+z\left(\bar{D} u_{1} \circ u_{2}+u_{1} \circ \bar{D} u_{2}\right)+z^{2} \bar{D} u_{1} \circ \bar{D} u_{2}\right) w \\
= & u_{1} \wedge u_{2} \wedge w+z \bar{D} u_{1}\left(u_{2} \wedge w\right)+u_{1} \wedge \bar{D} u_{2}(w)+z^{2} \bar{D} u_{1}\left(\bar{D} u_{2}(w)\right) \\
= & u_{1} \wedge u_{2} \wedge w+z\left(u_{2} \wedge D_{1} u_{1} \wedge w+u_{1} \wedge\left(w \wedge D_{1} u_{2}\right)\right)+z^{2} \bar{D} u_{1}\left(w \wedge D_{1} u_{2}\right) \\
= & u_{1} \wedge u_{2} \wedge w+z\left(u_{2} \wedge D_{1} u_{1}+D_{1} u_{2} \wedge u_{1}\right) \wedge w+z^{2}\left(w \wedge D_{1} u_{1} \wedge D_{1} u_{2}\right) \\
= & {\left[u_{1} \wedge u_{2}+\left(D_{1} u_{2} \wedge u_{1}+u_{2} \wedge D_{1} u_{1}\right) z+\left(D_{1} u_{1} \wedge D_{1} u_{2}\right) z^{2}\right] \wedge w . }
\end{aligned}
$$

We have obtained:

$$
\begin{gathered}
\bar{D}_{1}\left(u_{1} \wedge u_{2}\right)=\left(D_{1} u_{2} \wedge u_{1}+u_{2} \wedge D_{1} u_{1}\right) \wedge_{-}: \bigwedge V_{n} \rightarrow \bigwedge V_{n}, \\
\bar{D}_{2}\left(u_{1} \wedge u_{2}\right)=\left(D_{1} u_{1} \wedge D_{1} u_{2}\right) \wedge_{-}: \bigwedge V_{n} \rightarrow \bigwedge V_{n} .
\end{gathered}
$$

Similarly we can find that

$$
\bar{D}_{2}\left(u_{1} \wedge u_{2} \wedge u_{3}\right)=\bar{D}_{2}\left(u_{1} \wedge u_{2}\right) \circ u_{3}+\bar{D}_{1}\left(u_{1} \wedge u_{2}\right) \circ \bar{D}_{1} u_{3}
$$

i.e., more explicitly

$\bar{D}_{2}\left(u_{1} \wedge u_{2} \wedge u_{3}\right) \wedge w=D_{1} u_{1} \wedge D_{1} u_{2} \wedge u_{3} \wedge w+\left(D_{1} u_{2} \wedge u_{1}+u_{2} \wedge D_{1} u_{1}\right) \wedge w \wedge D_{1} u_{3}$ from which

$$
\begin{aligned}
\bar{D}_{2}\left(u_{1}\right. & \left.\wedge u_{2} \wedge u_{3}\right) \wedge w \\
& =\left(D_{1} u_{1} \wedge D_{1} u_{2} \wedge u_{3}+D_{1} u_{2} \wedge D_{1} u_{3} \wedge u_{1}+u_{2} \wedge D_{1} u_{3} \wedge D_{1} u_{1}\right) \wedge w \\
& =\left(D_{1} u_{1} \wedge D_{1} u_{2} \wedge u_{3}+u_{1} \wedge D_{1} u_{2} \wedge D_{1} u_{3}+D_{1} u_{1} \wedge u_{2} \wedge D_{1} u_{3}\right) \wedge w .
\end{aligned}
$$

We could say that the operators $\bar{D}_{1}\left(u_{1} \wedge u_{2}\right), \bar{D}_{2}\left(u_{1} \wedge u_{2}\right)$, and $\bar{D}_{2}\left(u_{1} \wedge u_{2} \wedge u_{3}\right)$ are "represented" respectively by the following elements of $\bigwedge V_{n}$ :

$$
D_{1} u_{2} \wedge u_{1}+u_{2} \wedge D_{1} u_{1}=D_{1}\left(u_{2} \wedge u_{1}\right), \quad D_{1} u_{1} \wedge D_{1} u_{2},
$$

and

$$
D_{1} u_{1} \wedge D_{1} u_{2} \wedge u_{3}+u_{1} \wedge D_{1} u_{2} \wedge D_{1} u_{3}+D_{1} u_{1} \wedge u_{2} \wedge D_{1} u_{3} .
$$

Remark 3.11. Let us check that $D_{1} u \wedge v+\left(\bar{D}_{1} u\right)(v) \succeq 0$, which is the sense we want to give to the expression $D_{1}+\bar{D}_{1} \succeq 0$. For all $u, v \in \wedge V_{n}$. If $u \in V_{n}$ and $v=v_{1} \wedge v_{2}$ with $v_{1} \in V_{n}$ :

$$
\begin{aligned}
D_{1} u \wedge v+\left(\bar{D}_{1} u\right)(v) & =\left(D_{1} u \wedge v_{1}\right) \wedge v_{2}+(\bar{D} u)\left(v_{1}\right) \wedge v_{2} \\
& =D_{1} u \wedge v_{1} \wedge v_{2}+v_{1} \wedge D_{1} u \wedge v_{2} \\
& =\left(D_{1} u \wedge v_{1}+v_{1} \wedge D_{1} u\right) \wedge v_{2} \succeq 0 .
\end{aligned}
$$

More in general we have the following crucial:

Theorem 3.12. The polynomial $\bar{D}\{z\}$ is a quasi-inverse of $D\{z\}$, in the sense that for all $u \in \bigwedge V_{n}$

$$
\bar{D}\{z\} D\{z\} u \succeq u .
$$


Proof. We first check that the property holds for all $u \in V_{n}$. Then, for all $w \in \bigwedge V_{n}$

$$
\begin{aligned}
(\bar{D}\{z\} D\{z\} u)(w) & =(D\{z\} u+z \bar{D} D\{z\} u)(w) \\
& =D\{z\} u \wedge w+z w \wedge D_{1} D\{z\} u \\
& =\left(u+z D_{1} D\{z\} u\right) \wedge w+z \cdot w \wedge D_{1} D\{z\} u \\
& =u \wedge w+z\left(D_{1} D\{z\} u \wedge w+w \wedge D_{1} D\{z\} u\right) \succeq u \wedge w
\end{aligned}
$$

for all $w \in \wedge V_{n}$. Thus we have proved that $\bar{D}\{z\} D\{z\} u \wedge_{-} \succeq u \wedge_{\text {, }}$, and the property is checked for all $u \in \bigwedge^{1} V_{n}$. Now, we argue by induction, by supposing the property holds true for all $u \in \bigwedge^{\leq k-1} V_{n}$. Let us prove it for all $u \in \bigwedge^{k} V_{n}$. In this case we can assume $u$ of the form $u_{1} \wedge v$, with $u_{1} \in V_{n}$. Then

$$
\bar{D}\{z\}\left(D\{z\}\left(u_{1} \wedge v\right)\right)=\bar{D}\{z\}\left(D\{z\} u_{1}\right) \circ \bar{D}\{z\}(\bar{D}\{z\} v) \succeq\left(u_{1} \wedge v\right) \wedge \_
$$

having used induction and the first step.

Counterexample 3.13. Quite surprisingly, while $\bar{D}\{z\}$ is a quasi-inverse of $D\{z\}$, the reverse is not true. For instance, for all $u, v \in V_{n}$ one has: $D\{z\}(\bar{D}\{z\} u)(v) \succeq$ $u \wedge D\{z\} v=u+D\{z\} D_{1} u$. Let us check it, recalling that if $u \in V_{n}$ then $D\{z\} u=$ $u+\sum_{i \geq 0} D_{1}^{i} u \cdot z^{i}$.

$$
\begin{array}{rlr}
D\{z\} & (\bar{D}\{z\} u)(v) & \\
& =D\{z\}\left(u \wedge v+z \cdot v \wedge D_{1} u\right) & \\
& =D\{z\} u \wedge D\{z\} v+z D\{z\} v \wedge D\{z\} D_{1} u & \text { (definition (3.7) of }(D\{z\} u)(v)) \\
& =u \wedge D\{z\} v+z D\{z\} D_{1} u \wedge D\{z\} v & \\
& +z D\{z\} v \wedge D\{z\} D_{1} u & \\
& \succeq u \wedge D\{z\} v . &
\end{array}
$$

As an additional check, notice that if $D\{z\}$ were a quasi-inverse of $\bar{D}\{z\}$, then

$$
\left(1+D_{1} z+D_{2} z^{2}+\cdot\right)\left(1+\bar{D}_{1}+\bar{D}_{2} z^{2}+\cdots\right) \succeq 0
$$

In particular, considering the coefficient of $z^{2}$ in both sides, the following surpassing relation should hold:

$$
D_{2}+D_{1} \bar{D}_{1}+\bar{D}_{2} \succeq 0 .
$$

But (3.11) already fails for $u \in V_{n}=\bigwedge^{1} V_{n}$. Indeed, for all $v \in V_{n}$, and noting that $\bar{D}_{2} u=0$, by Remark 3.9 .

$$
\begin{aligned}
\left(D_{2} u\right. & \left.+D_{1} \bar{D}_{1} u+\bar{D}_{2} u\right)(v) \\
& =D_{2} u \wedge v+D_{1}\left(v \wedge D_{1} u\right) \\
& =D_{2} u \wedge v+D_{1} v \wedge D_{1} u+v \wedge D_{2} u \\
& =D_{1} v \wedge D_{1} u+D_{2} u \wedge v+v \wedge D_{2} u \\
& \succeq D_{1} v \wedge D_{1} u
\end{aligned}
$$

Theorem 3.14. $\bar{D}\{z\}(D\{z\} u \wedge v) \succeq u \wedge \bar{D}\{z\} v, \quad \forall u, v \in \wedge V_{n}$.

Proof. Suppose $u, v$ are homogeneous, say $u \in \bigwedge^{k} V_{n}$ and $v \in \bigwedge^{\ell} V_{n}$. Then, by Definition 3.8 of $\bar{D}\{z\}$,

$$
\bar{D}\{z\}(D\{z\} u \wedge v)=\bar{D}\{z\}(D\{z\} u) \circ \bar{D}\{z\} v
$$


By Theorem 3.12, $\bar{D}\{z\}(D\{z\} u \wedge v)(w) \succeq u \circ(\bar{D}\{z\} v)(w)=u \wedge(\bar{D}\{z\} v)(w)$, because $u$ acts as an endomorphism on vectors of $\bigwedge V_{n}$ as $u \wedge$, for all $w \in \Lambda V_{n}$.

3.2. The Cayley-Hamilton formulas for semialgebras. Formally define $\zeta=$ $b_{0} \wedge b_{1} \wedge \cdots \wedge b_{n-1}$ and $\zeta^{\prime}=b_{1} \wedge b_{0} \wedge \cdots \wedge b_{n-1}$. Thus $\zeta^{\prime}=(-) \zeta$, and

$$
\bar{D}_{i} \zeta=e_{i} \zeta+e_{i}^{\prime} \zeta^{\prime}, \quad e_{i}, e_{i}^{\prime} \in \mathcal{A} .
$$

In other words, $\left(e_{i}, e_{i}^{\prime}\right)$ could be called the eigenvalue pair of $\bar{D}_{i}$ restricted to $\bigwedge^{n} V_{n}$ (where in some sense $e_{i}^{\prime}$ is the negated part). Let $E_{n}(z)$ be the eigenvalue polynomial of $\bar{D}\{z\}$, i.e.

$$
E_{n}(z) \zeta:=\bar{D}\{z\} \zeta+\bar{D}\{z\} \zeta^{\prime}=\left(1+e_{1} z+\cdots+e_{n} z^{n}\right) \zeta+\left(1+e_{1}^{\prime} z+\cdots+e_{n}^{\prime} z^{n}\right) \zeta^{\prime} .
$$

In particular if one sets $D_{i} \zeta=h_{i} \zeta+h_{i}^{\prime} \zeta^{\prime}$, the relations $\bar{D}\{z\} D\{z\} \zeta \succeq \zeta$ and $\bar{D}\{z\} D\{z\} \zeta^{\prime} \succeq \zeta^{\prime}$ yield the relation

$$
\left(h_{n}+e_{1} h_{n-1}+\cdots+e_{n}\right)+\left(h_{n}^{\prime}+e_{1}^{\prime} h_{n-1}^{\prime}+\cdots+e_{n}^{\prime}\right) \succeq \mathbb{0} .
$$

Theorem 3.15. The Cayley-Hamilton formulas

$$
\left(\left(D_{n} u+e_{1} D_{n-1} u+\cdots+e_{n} u\right) \wedge v\right)(-)\left(\left(D_{n} u+e_{1}^{\prime} D_{n-1} u+\cdots+e_{n}^{\prime} u\right) \wedge v\right) \succeq \mathbb{0}
$$

hold for all $u \in \bigwedge^{>0} V_{n}$, i.e., the left side is a quasi-zero.

Proof. If $u=\zeta$ the theorem is true, due to (3.13). Then assume that $u \in \bigwedge^{n-i} V_{n}$, for some $1 \leq i \leq n-1$. This follows from the transfer principle of Remark 1.7. since the assertion was proved (with equality) for classical algebras in [6, and all the extra quasi-zeros appear in the right. But we also would like to give a direct proof. For all $v \in \bigwedge^{i} V_{n}$ we have the surpassing relation (3.14). Matching degrees yields the surpassing relation between the $n$-th degree coefficient of the left side and the $n$-th degree coefficient of the right side of (3.14) which is:

$$
D_{n} u \wedge v+\bar{D}_{1}\left(D_{n-1} u \wedge v\right)+\cdots+\bar{D}_{n}(u \wedge v) \succeq u \wedge \bar{D}_{n} v .
$$

Since $\bar{D}\{z\} v$ is a polynomial of degree at most $i<n$, it follows that $\bar{D}_{k} v \succeq \mathbb{0}$ for all $k>i$. On the other hand $\bar{D}_{i}\left(D_{n-i} u \wedge v\right)=e_{i}\left(D_{n-i} u \wedge v\right)(-) e_{i}^{\prime}\left(D_{n-i} u \wedge v\right)$ because $\left(e_{i}, e_{i}^{\prime}\right)$ is the eigenvalue pair of $\bar{D}_{i}$ against any element of $\bigwedge^{n} V_{n} \cong\left(\mathcal{A} \zeta+\mathcal{A} \zeta^{\prime}\right)$. Thus we have proved (3.14) for all $v \in \bigwedge V_{n}$.

Remark 3.16. In the classical case where the only quasi-zero is $\{\mathbb{O}\}$, we get

$$
\left(\left(D_{n} u+e_{1} D_{n-1} u+\cdots+e_{n} u\right) \wedge v\right)(-)\left(\left(D_{n} u+e_{1}^{\prime} D_{n-1} u+\cdots+e_{n}^{\prime} u\right) \wedge v\right)=\mathbb{O} .
$$

Corollary 3.17. $\left(D_{1}^{n}+\left(e_{1}(-) e_{1}^{\prime}\right) D_{1}^{n-1}+\cdots+\left(e_{n}(-) e_{n}^{\prime}\right)\right) u \succeq \mathbb{O}$ for all $u \in \bigwedge^{>0} V_{n}$, where we interpret $\left(e_{i}(-) e_{i}^{\prime}\right) D_{i}^{n-i}(u)$ to be $e_{i} D_{i}^{n-i} u(-) e_{i}^{\prime} D_{i}^{n-i} u$.

Proof. By Theorem 3.15

$$
\left(\left(D_{n} u+e_{1} D_{n-1} u+\cdots+e_{n} u\right) \wedge v\right)(-)\left(\left(D_{n} u+e_{1}^{\prime} D_{n-1} u+\cdots+e_{n}^{\prime} u\right) \wedge v\right) \succeq \mathbb{0} .
$$

But $D\{z\}$ is by hypothesis the unique HS-derivation on $\bigwedge V_{n}$ associated to the endomorphism $D_{1}$ (see Proposition 3.4). In particular $D_{i} u=D_{1}^{i} u$.

Note 3.18. When working with $\mathfrak{G}(V)_{\geq 2}$, we obtain equality in Theorem 3.15 and Corollary 3.17 since the only quasi-zeros are $\mathbb{0}$, by Theorem 2.9 


\section{REFERENCES}

[1] Marianne Akian, Stéphane Gaubert, and Alexander Guterman, Tropical Cramer determinants revisited, Tropical and idempotent mathematics and applications, Contemp. Math., vol. 616, Amer. Math. Soc., Providence, RI, 2014, pp. 1-45, DOI 10.1090/conm/616/12324. MR 3221324

[2] George M. Bergman, The diamond lemma for ring theory, Adv. in Math. 29 (1978), no. 2, 178-218, DOI 10.1016/0001-8708(78)90010-5. MR506890

[3] Letterio Gatto, Schubert calculus via Hasse-Schmidt derivations, Asian J. Math. 9 (2005), no. 3, 315-321, DOI 10.4310/AJM.2005.v9.n3.a2. MR2214955

[4] Letterio Gatto and Parham Salehyan, Hasse-Schmidt derivations on Grassmann algebras: With applications to vertex operators, IMPA Monographs, vol. 4, Springer, [Cham], 2016. MR.3524604

[5] L. Gatto and I. Scherbak, On generalized Wronskians, Contributions in Algebraic Geometry, Impanga Lecture Notes (P. Pragacz Ed.), EMS Congress Series Report, 257-296, 2012, available at http://xxx.lanl.gov/pdf/1310.4683.pdf.

[6] Letterio Gatto and Inna Scherbak, Hasse-Schmidt derivations and Cayley-Hamilton theorem for exterior algebras, Functional analysis and geometry: Selim Grigorievich Krein centennial, Contemp. Math., vol. 733, Amer. Math. Soc., Providence, RI, 2019, pp. 149-165, DOI 10.1090/conm/733/14739. MR3985273

[7] S. Gaubert, Théorie des systèmes linéaires dans les diodes, Thèse, École des Mines de Paris, 1992.

[8] Jeffrey Giansiracusa and Noah Giansiracusa, A Grassmann algebra for matroids, Manuscripta Math. 156 (2018), no. 1-2, 187-213, DOI 10.1007/s00229-017-0958-z. MR3783573

[9] Jonathan S. Golan, The theory of semirings with applications in mathematics and theoretical computer science, Pitman Monographs and Surveys in Pure and Applied Mathematics, vol. 54, Longman Scientific \& Technical, Harlow; copublished in the United States with John Wiley \& Sons, Inc., New York, 1992. MR1163371

[10] F. K. Schmidt and H. Hasse, Noch eine Begründung der Theorie der höheren Differentialquotienten in einem algebraischen Funktionenkörper einer Unbestimmten. (Nach einer brieflichen Mitteilung von F.K. Schmidt in Jena) (German), J. Reine Angew. Math. 177 (1937), 215-237, DOI 10.1515/crll.1937.177.215. MR1581557

[11] Nickolas Heerema, Derivations and embeddings of a field in its power series ring, Proc. Amer. Math. Soc. 11 (1960), 188-194, DOI 10.2307/2032953. MR 123568

[12] Nickolas Heerema, Derivations and embeddings of a field in its power series ring. II, Michigan Math. J. 8 (1961), 129-134. MR136601

[13] Nickolas Heerema, Higher derivations and automorphisms of complete local rings, Bull. Amer. Math. Soc. 76 (1970), 1212-1225, DOI 10.1090/S0002-9904-1970-12609-X. MR266916

[14] Zur Izhakian, Manfred Knebusch, and Louis Rowen, Supertropical quadratic forms I, J. Pure Appl. Algebra 220 (2016), no. 1, 61-93, DOI 10.1016/j.jpaa.2015.05.043. MR.3393451

[15] J. Jun, K. Mincheva, and L. Rowen, Homology of module systems, arXiv:1809.01996, J. Pure Appl. Algebra, to appear (2020).

[16] Jaiung Jun and Louis Rowen, Categories with negation, Categorical, homological and combinatorial methods in algebra, Contemp. Math., vol. 751, Amer. Math. Soc., Providence, RI, [2020] (C)2020, pp. 221-270, DOI 10.1090/conm/751/15120. MR/4132092

[17] E. B. Kacov, The tensor product of functors (Russian), Sibirsk. Mat. Zh. 19 (1978), no. 2, 318-327, 478. MR 489991

[18] Yefim Katsov, Tensor products and injective envelopes of semimodules over additively regular semirings, Algebra Colloq. 4 (1997), no. 2, 121-131. MR1682765

[19] Oliver Lorscheid, The geometry of blueprints: Part I: Algebraic background and scheme theory, Adv. Math. 229 (2012), no. 3, 1804-1846, DOI 10.1016/j.aim.2011.12.018. MR2871157

[20] M. H. A. Newman, On theories with a combinatorial definition of "equivalence.", Ann. of Math. (2) 43 (1942), 223-243, DOI 10.2307/1968867. MR7372

[21] L. H. Rowen, Algebras with a negation map, 75 pages, arXiv:1602.00353 [math.RA].

[22] Louis Rowen, An informal overview of triples and systems, Rings, modules and codes, Contemp. Math., vol. 727, Amer. Math. Soc., Providence, RI, 2019, pp. 317-335, DOI 10.1090/conm/727/14644. MR.3938159 
[23] Michihiro Takahashi, On the bordism categories. I, Math. Sem. Notes Kobe Univ. 8 (1980), no. 3, 527-546. MR615872

[24] Wikipedia, Exponential of nilpotent derivation with divided Leibniz condition powers is endomorphism.

Dipartimento di Scienze Matematiche, Politecnico di Torino, C. So Duca degli Abruzzi 24, 10129 Torino - ItAlia

Email address: letterio.gatto@polito.it

Department of Mathematics, Bar-Ilan University, Ramat-Gan 52900, Israel

Email address: rowen@math.biu.ac.il 